EPJ Web of Conferences 16, 05006 (2011)

DOI: $10.1051 /$ epjconf/20111605006

(C) Owned by the authors, published by EDP Sciences, 2011

\title{
The grain dynamics and structures of debris discs
}

\author{
L.-C. Yeh ${ }^{1, a}$ and I.-G. Jiang ${ }^{2, b}$ \\ ${ }^{1}$ Department of Applied Mathematics, National Hsinchu University of Education, Hsin-Chu, \\ Taiwan \\ ${ }^{2}$ Department of Physics and Institute of Astronomy, National Tsing-Hua University, Hsin-Chu, \\ Taiwan
}

\begin{abstract}
Numerical simulations are used to construct the possible dynamical models of the Vega's debris disc. New dust grains are assumed to be generated from two clumpy regions. The goal is to test whether an overall circular-symmetric structure can be constructed if the birth places of grains are not uniformly distributed in a ring region.
\end{abstract}

\section{INTRODUCTION}

Confirmed by the infrared excesses, circumstellar dust exists around many main sequence stars. It is an interesting question that whether these dust grains are primordial or generated after the formation of star-disc systems. Recently, the observational work shows that the dust grains could be from the collision of planetesimals, but the possibility that they are the protoplanetary disc remnants cannot be excluded. The fact could be that both sources are important because certain amount of dust grains should remain in the protoplanetary disc during the star formation process, and the generation of new grains definitely takes place as many small grains would be blown away by the radiation pressure continuously.

In the standard scenario, debris discs are constructed at the time when planetesimals are forming and colliding frequently. Thus, debris discs can be generated only when there are km-sized planetesimals colliding and producing huge amount of new dust grains. This would take place at the stellar age of million years when the original seed grains grow to become km-sized planetesimals Cuzzi et al. (1993). Moreover, in addition to creating new dust grains, the planetesimals would further grow into asteroids and also trigger the formation of planets. In the end, the gaseous parts are gradually depleted by the stellar wind and the debris discs are constructed.

Therefore, due to that it is necessary to generate new grains frequently, the existence of a debris disc implies the presence of planetesimals and probably also planets. The study of debris discs is thus very interesting and important because the density structures and evolutionary histories of debris discs actually provide hints on the evolution of planetesimals and the formation of planets.

Since the Vega system gives one of the closest and brightest debris discs, many observations have been done and revealed the detail information. Su et al. (2005) show images of Vega observed by the Spitzer Space Telescope, and claim a ring region, from $86 \mathrm{AU}$ to $200 \mathrm{AU}$, keeps producing new grains, which migrate outward and form $1 / R$ density profile of the outer disc.

ae-mail: lcyeh@mail.nhcue.edu.tw

This is an Open Access article distributed under the terms of the Creative Commons Attribution-Noncommercial License 3.0, which permits unrestricted use, distribution, and reproduction in any noncommercial medium, provided the original work is properly cited. 

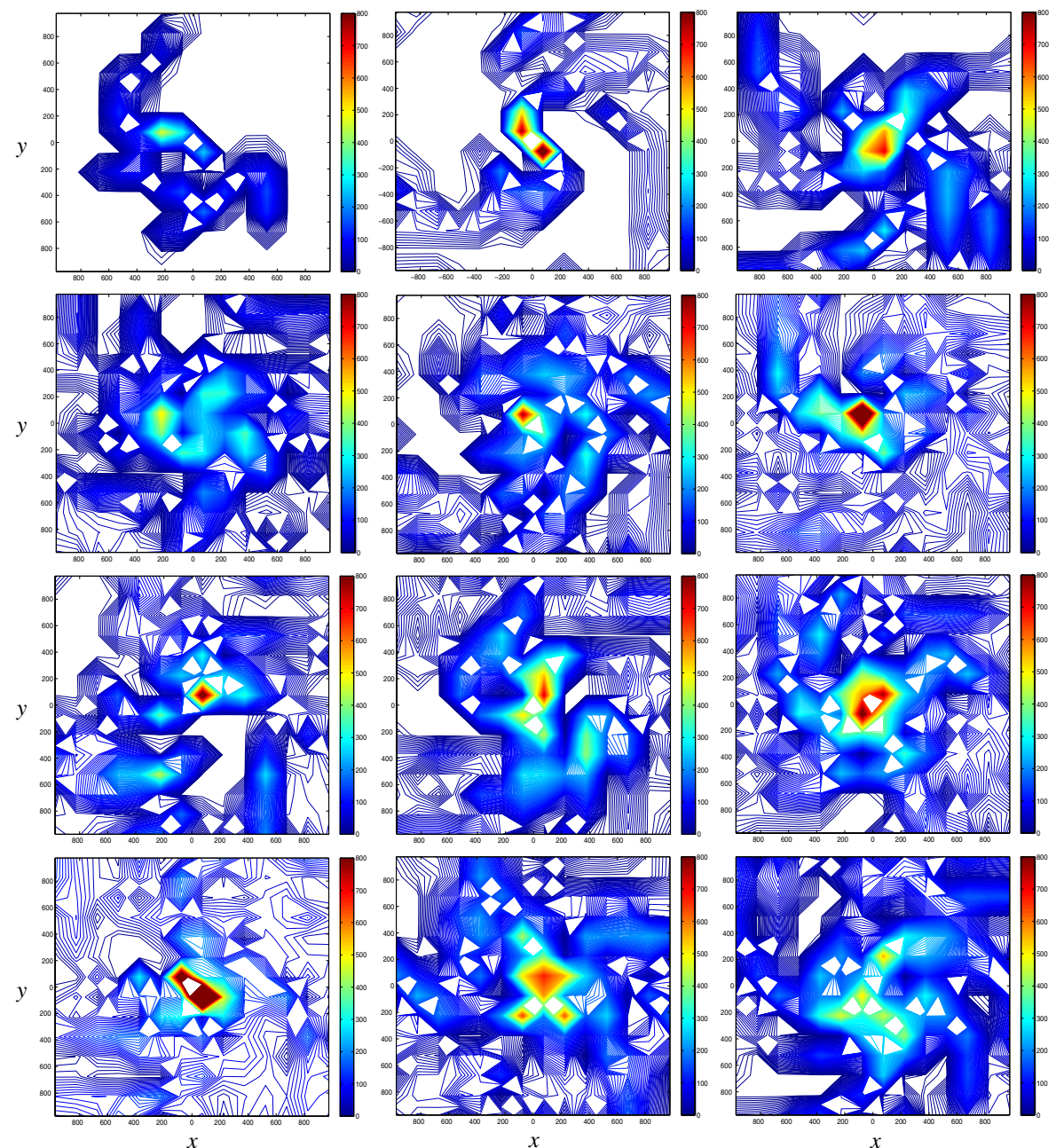

Figure 1. The surface mass density of a debris disc in the simulation with $t_{p}=20$. The time difference between panels is 1000 years.

\section{THE VEGA PUZZLE}

For such a blowing-out picture, it would need a huge amount of mass if it starts right after the Vega system was formed.Su et al. (2005) therefore claim small dust grains are from a recent large collisional event.

However, if there was a recent large collisional event, the majority of new dust grains shall be generated at a small area in the inner part of the debris disc. This shall remain to be true even the subsequent collisional cascade are taken into account.

It is a puzzle that the face-on image of Vega's debris disc looks as a complete circular-symmetric structure, as shown in Su et al. (2005). The problem can not be solved even the spacial resolution of the telescope is considered.

Assuming that the dust grains are generated uniformly from a ring area, Jiang \& Yeh (2009) used numerical simulations to construct the possible dynamical models of the Vega's outer disc. The grains' orbits are calculated and the surface densities of the outer discs are determined in these models. Their 
Research, Science and Technology of Brown Dwarfs and Exoplanets

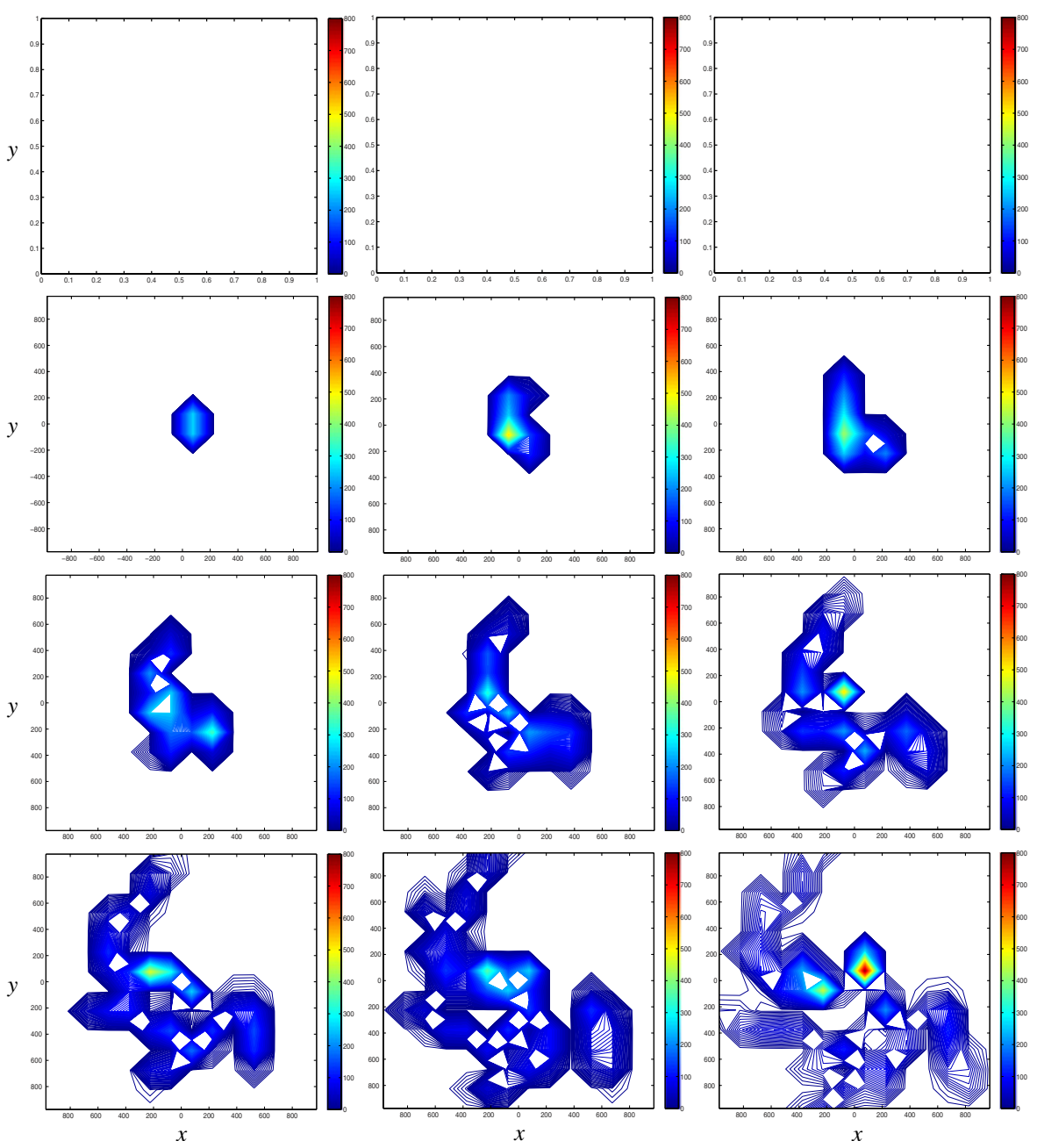

Figure 2. The surface mass density of a debris disc in the simulation with $t_{p}=20$. The time difference between panels is 100 years.

results show that the blowing-out dynamical models can be constructed given that the small grains are produced frequently enough. In this paper, we decide to do simulations that the grains are generated from two clumps of high density regions only. In this way, we hope to check whether the overall circularsymmetric structure can be constructed if the birth places of grains are not uniformly distributed in a ring region.

\section{THE MODEL}

We assume that there is a planet moving on a circular orbit with radius $R=54 \mathrm{AU}$. As the $3: 2$ resonance region is around $R=70 \mathrm{AU}$, two clumps with higher surface mass densities of planetesimals are set to be centered at $(0,70)$ and $(-70,0)$ initially, and continuously move together with the planet by the same angular speed. To simplify the model, we assume both clumps to be circular with radius $10 \mathrm{AU}$. 

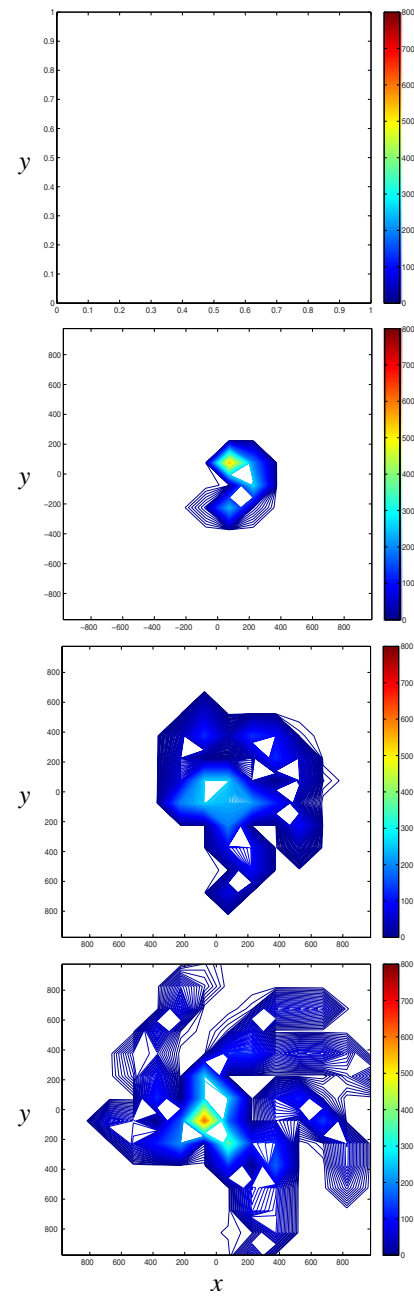
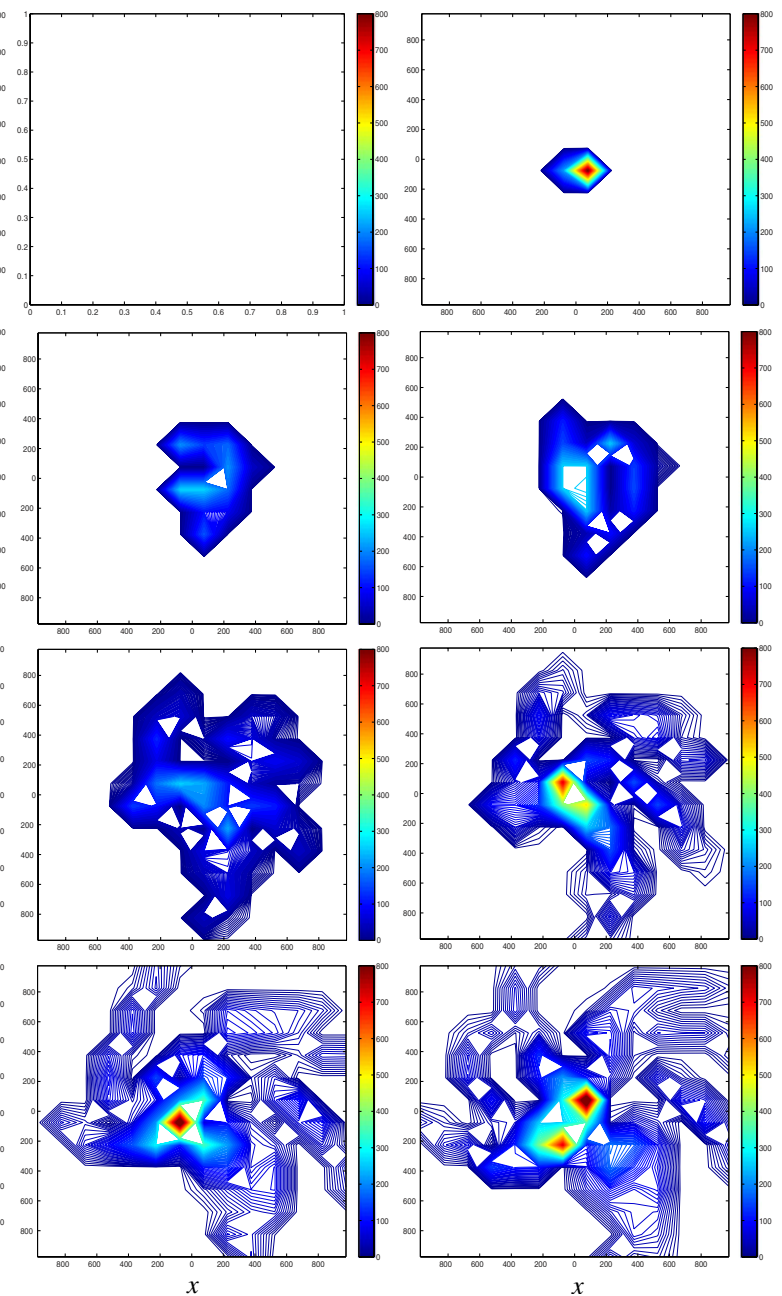

Figure 3. The same as in Fig. 2, except $t_{p}=10$.

In addition, there are 200 planetesimals uniformly distributed in a ring region between $R=60$ and 80 AU initially. They are not resonant particles, but simply move on Keplerian circular orbits. They are assumed to be massive enough and feel no influence from the radiation pressure.

Every $t_{p}$ years $\left(t_{p}=10\right.$ or 20$)$, all planetesimals located inside the clumps would be given a probability of $10 \%$ to collide with background unseen planetesimals, and produce new dust grains. The rest of model details are based on the method in Jiang \& Yeh (2009).

\section{THE RESULTS AND CONCLUDING REMARKS}

Figure 1 shows the surface mass density of a debris disc in our simulation with $t_{p}=20$. The topleft panel is for $t=1000$ years, and the one next to it is for $t=2000$ years etc. The arm and clumpy structures are developed gradually. In order to see the situation at the early stage for the same simulation, in Figure 2, the top-left panel is plotted for $t=100$ years, and the time difference between panels is 100 years. To make the collisions happen more often, we run another simulation with $t_{p}=10$ and the results are shown in Figure 3. 
Research, Science and Technology of Brown Dwarfs and Exoplanets

We conclude that when the collisions happen more often, it is possible that the debris disc becomes more circular-symmetric. However, it is unclear whether the overall collision probability and the needed mass is well justified. We will do more simulations to investigate this issue in the near future.

\section{References}

Cuzzi, J. N., Dobrovolskis, A. R., Champney, J. M. 1993, Icarus, 106, 102

Jiang, I.-G., Yeh, L.-C. 2009, AJ, 137, 4169

Su, K. Y. L. et al. 2005, ApJ, 628, 487 